\title{
12 Two-Dimensional Shape Coding
}

\author{
Joern Ostermann and Anthony Vetro
}

\section{CONTENTS}

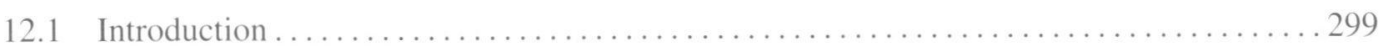

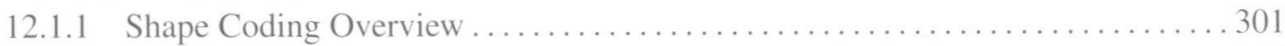

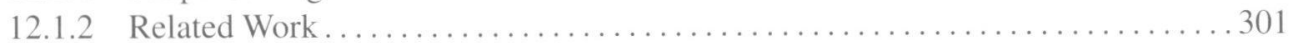

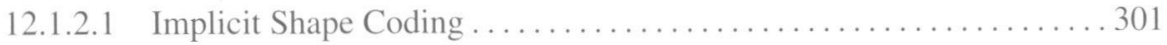

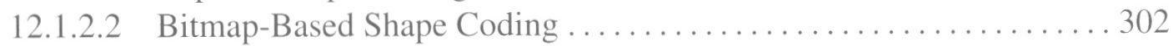

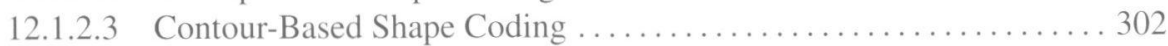

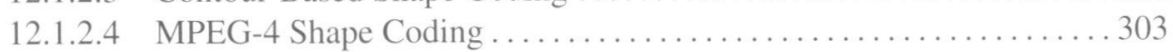

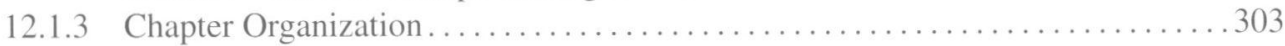

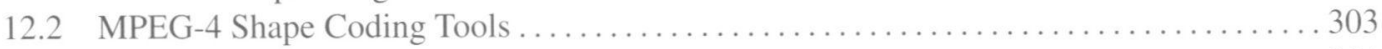

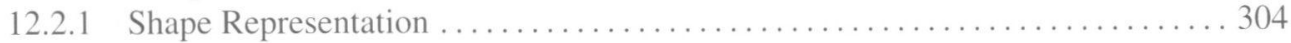

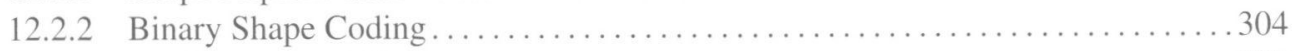

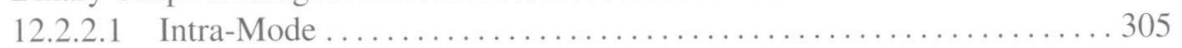

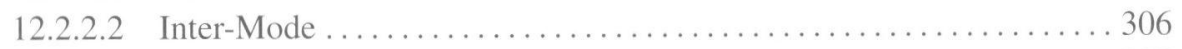

12.2.2.3 Evaluation Criteria for Coding Efficiency .................. 307

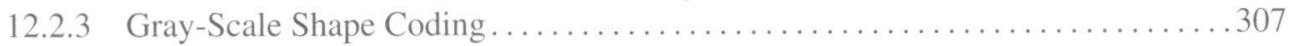

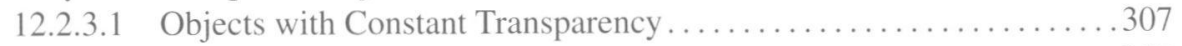

12.2.3.2 Objects with Arbitrary Transparency . . . . . . . . . . . . . . . . 307

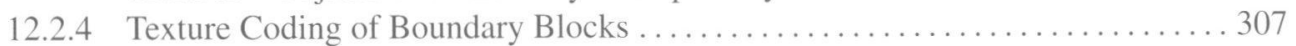

12.2.5 Video Coder Architecture . . . . . . . . . . . . . . . . . . . . . . . . . . . 308

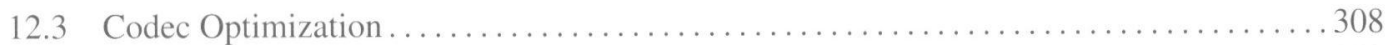

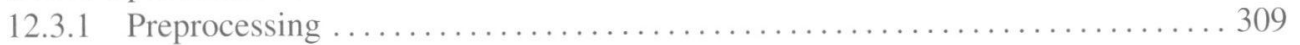

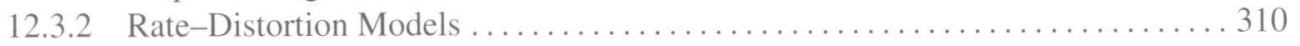

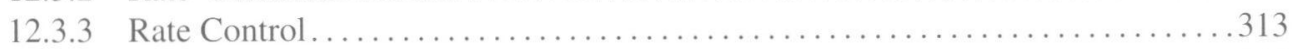

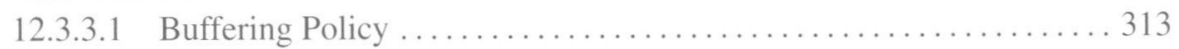

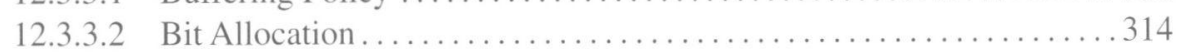

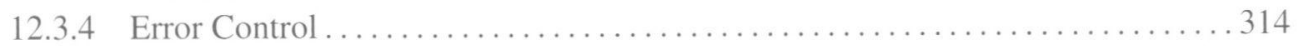

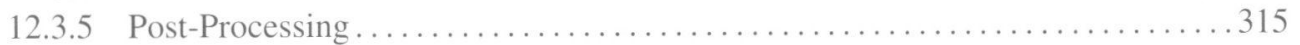

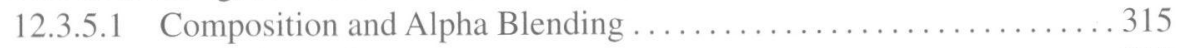

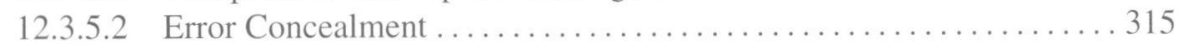

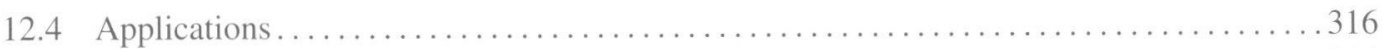

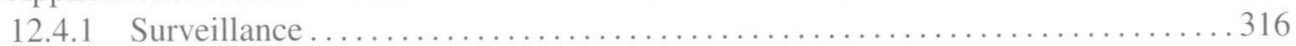

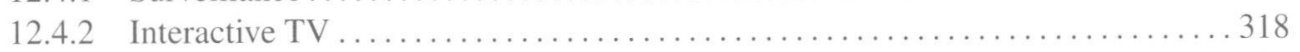

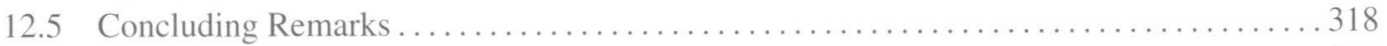

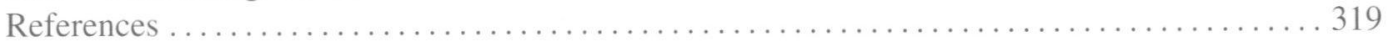

\subsection{INTRODUCTION}

With video being a ubiquitous part of modern multimedia communications, new functionalities in addition to the compression as provided by conventional video coding standards like H.261, MPEG-1, H.262, MPEG-2, H.263, and H.264 are required for new applications. Applications like 
content-based storage and retrieval have to allow access to video data based on object descriptions, where objects are described by texture, shape, and motion. Studio and television postproduction applications require editing of video content with objects represented by texture and shape. For collaborative scene visualization like augmented reality, we need to place video objects into the scene. Mobile multimedia applications require content-based interactivity and content-based scalability in order to allocate limited bit rate or limited terminal resources to fit the individual needs. Security applications benefit from content-based scalability as well. All these applications share one common requirement: video content has to be easily accessible on an object basis. MPEG-4 Visual enables this functionality. The main part of this chapter describes MPEG-4 shape coding, the content-based interactivity enabling tool

Given the application requirements, video objects have to be described not only by texture, but also by shape. The importance of shape for video objects has been realized early on by the broadcasting and movie industries employing the so-called chroma-keying technique, which uses a predefined color in the video signal to define the background. Coding algorithms like objectbased analysis-synthesis coding (OBASC) [30] use shape as a parameter in addition to texture an motion for describing moving video objects. Second gentration image coding segments an image into regions and describes each region by texture and shape $[28]$. The purpose of using shape was achieve better subjective picture quality, increased coding efficiency as well as an object-based video representation.

MPEQ-4 Visual is the first international standard allowing the transmission of arbitrarily shaped video objects (VO) [21]. Each frame of a VO is called video object plane (VOP) consisting of shape and texture information as well as optional motion information. Following an object-based approach, MPEG-4 Visual transmits texture, motion, and shape information of one VO within on bitstream. The bitstreams of several VOs and accompanying composition information can be mutiplexed such that the decoder receives all the information to decode the VOs and arrange then into a video scene; the composition of multiple video objects is ilfustrated in Figure 12.1. Alternatively, objects may be transmitted in different streams according to a scene description $[11,44$ This results in a new dimension of interactivity and flexibility for standardized video and multimedia applications.

Two types or VOs are distinguished. For opaque objects, binary shape information is transmitted. Transparent objects are described by gray-scale $\alpha$-maps defining the outline as well as the transparency variation of an object.

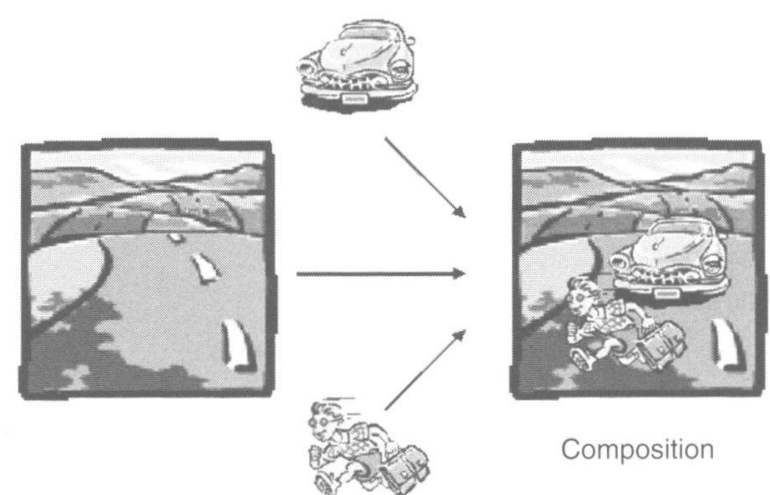

HGURE 12.1 Content-based scalability requires individual objects to be transmitted and composited at the decoder. Depending on resources, only some of the objects might be composited to the scene and presented the terminal.

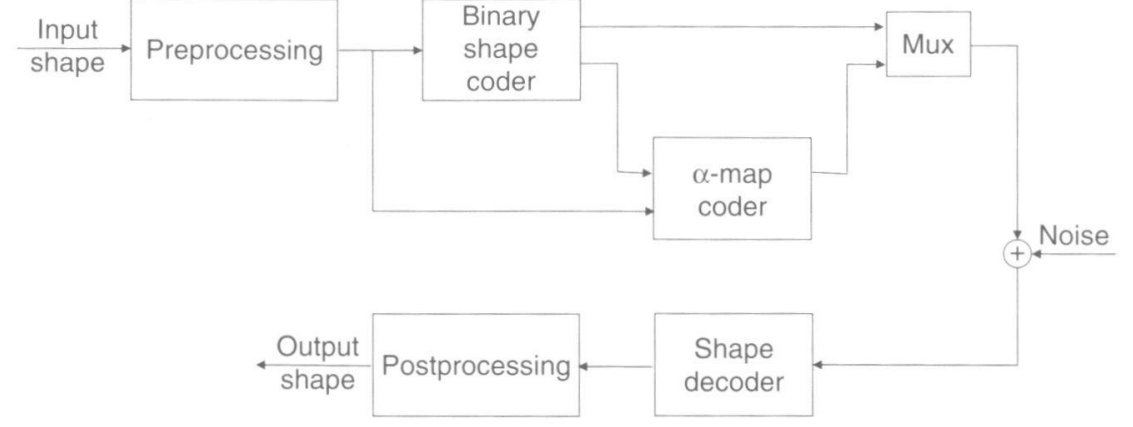

FIGURE 12.2 Processing steps for shape coding considering binary and gray-scale $\alpha$-maps.

\subsubsection{Shape Coding Overview}

Figure 12.2 shows the processing steps related to shape coding. They apply to object-based coding systems that transmit shape information only, as well as to systems that transmit texture for the objects. The optional shape preprocessing may remove noise from the shape signal and simplify the shapes such that it can be coded more efficiently. Preprocessing usually depends on the shape coding algorithm employed.

For transparent objects, the preprocessed shape information is separated into a binary shape defining the pels belonging to the object and a gray-scale information defining the transparency of each pel of the object. For binary shapes and gray-scale shape information the binary shape coder codes the shape using lossless or lossy shape coding algorithms. In the case of transparent objects, an $\alpha$-map coder codes the transparency information for the coded binary shape. The bitstreams get multiplexed, transmitted, and decoded at the decoder. The optional postprocessing algorithm provides error concealment and boundary smoothing.

The receiver decodes the VOs and composes them into a scene as defined by the composition information [11.44]. Typically, several VOs are overlayed on a background. For some applications a complete background image does not exist. Foreground VOs are used to cover these holes. Ofter they exactly fit into holes of the background. In case of lossily coded shape, a pixel originally defined as opaque may be changed to transparent thereby resulting in undefined pixels in the scene. Therefore, lossy shape coding of the background needs to be coordinated with lossy shape coding of the foreground VOs. If objects in a scene are not coded at the same temporal rate and a ful rectangular background does not exist, then it is very likely that undefined pixels in the scene will occur. Postprocessing in the decoder may extend objects to avoid these holes.

\subsubsection{Related Work}

There are three classes of binary shape coders. A bitmap-based coder encodes for each pel whether it belongs to the object or not. A contour-based coder encodes the outline of the object. In order to retrieve the bitmap of the object shape, the contour is filled with the object label. In the case where there is also texture transmitted with the shape information, an implicit shape coder, often referred to as chroma keying [7], can be used, where the shape information is derived from the texture using a predefined color for defining the outside of an object. Similar to texture coding, binary shapes can be coded in a lossless or lossy fashion.

\subsubsection{Implicit Shape Coding}

This class of coding defines a specific pixel value or a range of pixel values as the background of the image. The remaining pels are part of the object. An implicit shape coder is also specified in 
GIF89a [9]. For each image, one number can be used to define the value of the transparent pels. All pels of this value are not displayed. Today, GIF89a is used in web applications to describe arbitrarily shaped image and video objects.

\subsubsection{Bitmap-Based Shape Coding}

Such coding schemes are used in the fax standards G4 [4] and JBIG [19]. The modified read (MR) and encodes the location of changing pels where the scanline changes its color. In this line-by-line scheme, the position of each changing pel on the current line is coded with respect to either the position of a corresponding changing pel in the reference line, which lies immediately above the present line, or with respect to the preceding changing pel in the current line [31].

\subsubsection{Contour-Based Shape Coding}

Algorithms for contour-based coding and the related contour representations have been published extensively. Different applications nurtured this research: for lossless and lossy encoding of object boundaries, chain coders $[12,14]$ and polygon approximations $[15,18,34,42]$ were developed. For recognition purposes, shape representations like Fourier descriptors were developed to allow translation, rotation, and scale-invariant shape representations [55].

A chain code follows the contour of an object and encodes the direction in which the next boundary pel is located. Algorithms differ by whether they consider a pel having four or eight neighbors for rectangular grids or six neighbors for hexagonal grids. Some algorithms define the object boundary between pels [41]. Freeman [14] originally proposed the use of chain coding for boundary quantization and encoding, which has attracted considerable attention over the last 30 years $[23,27,32,38,39]$. The curve is quantized using the grid intersection scheme [14] and the quantized curve is represented using a string of increments. Since the planar curve is assumed to be continuous, the increments between grid points are limited to the eight grid neighbors, and hence an increment can be represented by 3 bits. For lossless encoding of boundary shapes, an average 1.2 to $1.4 \mathrm{bits} / \mathrm{boundary}$ pel are required [12]. There have been many extensions to this basic scheme such as the generalized chain codes [39], where the coding efficiency has been improved by using links of different length and different angular resolution. In [23], a scheme is presented which utilizes patterns in a chain code string to increase the coding efficiency and in [38], differential chain codes are presented, which employ the statistical dependency between successive links. There has also been interest in the theoretical performance of chain codes. In [27], the performance of different quantization schemes is compared, whereas in [32], the rate-distortion characteristics of certain chain codes are studied. Some chain codes also include simplifications of the contour in order to increase coding efficiency [33,37]. This is similar to filtering the object shape with morphological filters and then coding with a chain code [35]. The entropy coder may code a combination of several directions with just one code word.

A polygon-based shape representation was developed for OBASC $[17,18]$. As a quality measure, the maximum Euclidean distance $d_{\max }$ between the original and the approximated contour is used During subjective evaluations of CIF $(352 \times 288$ pels) video sequences, it was found that allowing a peak distance of $d_{\max }^{*}=1.4$ pel is sufficient to allow proper representations of objects in low bit-rate applications. Hence the lossy polygon approximation was developed. Vertices of the spline approximation do not need to be located on the object boundary [24,26].

This polygon representation can be also used for coding shapes in inter mode. For temporal prediction, the texture motion vectors are applied to the vertices of the previously coded shape defining the predicted shape for the current shape. Then, all vertices within the allowable approximation error $d_{\max }^{*}$ define the new polygon approximation. It is refined as described above such that the entire polygon is within the allowable error $d_{\max }^{*}$
In [22], B-spline curves are used to approximate a boundary. An optimization procedure is formulated for finding the optimal locations of the control points by minimizing the mean-squared error between the boundary and the approximation. In [24], a polygon/spline representation is described which provides optimality in the operational rate distortion sense.

Fourier descriptors describing the contour of an object were developed for applications in ecognition, where shape is an important key. Fourier descriptors allow translation, rotation, and scale-invariant representation [36]. In the first step, the coordinates of the contour are sampled clockwise in the $x y$-plane. This list of $2 \mathrm{D}$ coordinates $\left(x_{i}, y_{i}\right)$ is then transformed into an ordered list $\left(i,\left(y_{i+1}-y_{i} / x_{i+1}-x_{i}\right)\right)$ with $0 \leq i \leq i+1$ being the contour point number and $\left(y_{i+1}-y_{i} / x_{i+1}-x_{i}\right)$ being the change in direction of the contour. Since the samples are periodic over the object boundary perimeter, they can be expanded into a Fourier series. In order to preserve the main characteristics of a shape, only the large Founier coeficients have to be maintaned. Fourier descriptors are not very efficient in reconstruting polygon-like shapes with only a few coefficients. This is one of the reasons, why they never became very competitive in coding efficiency.

\subsubsection{MPEG-4 Shape Coding}

The MPEG committee investigated implicit, bitmap-based, and contour-based shape coding techniques. Implicit shape coding requires high-quality texture coding in order to derive a high-quality shape. Since humans tend to be more sensitive to shape distortions than to texture distortions, this coupling of shape and texture distortion is not acceptable for many applications. Therefore, while many different approaches for shape coding were evaluated during the development of MPEG-4, proposals for polygon-based contour coding and binary context-adaptive arithmetic coding were the lead contenders. Ultimately, MPEG-4 adopted the binary context-adaptive arithmetic coding, which is elaborated further in the next section.

The publication of the MPEG-4 standard and its work on shape coding [3,26,33] inspired the invention of many shape coding algorithms that are able to outperform MPEG-4 shape coding in terms of coding efficiency while being more computationally demanding. Mainly due to rate distortion optimization, the vertex-based method described in [24] provides an average rate reduction of 7.8\% with respect to the content-based arithmetic coder in MPEG-4. The skeleton-based method proposed in [54] gives bit rates 8 to $18 \%$ smaller than the MPEG-4 shape coder. In [1], digital straight lines are identified on the contour. These lines are then used to align a template for defining the context of an adaptive arithmetic encoder with the line. Although this algorithm only codes shapes in intra-mode, it requires 33\% less bits than the MPEG-4 shape coder in inter-mode as described in Section 12.2.

\subsubsection{Chapter Organization}

This chapter provides an overview of the algorithms for shape coding in MPEG-4. In Section 12.2, binary and gray-scale shape coding techniques are first described. Then, in Section 12.3, a variety of issues related to the encoding, modeling, and postprocessing of shape are discussed. Section 12.4 presents a few promising application that rely on shape coding, and finally, Section 12.5 presents

\subsection{MPEG-4 SHAPE CODING TOOLS}

This section will discuss the coding techniques for 2D shapes using MPEG- 4 tools. First, we briefly introduce the general representation for the 2D shape of an object, including binary and gray-scale hape. Then, we describe techniques for coding each of these representations. 

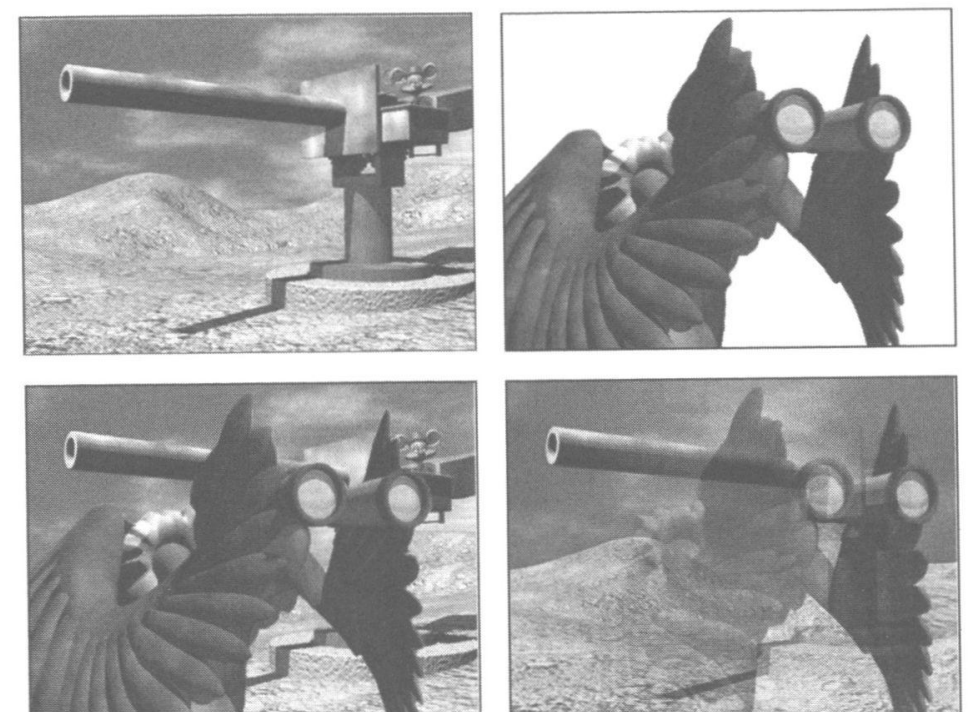

FIGURE 123 Comparison of scene composed with background image and foreground object with constant an gray-scale rransparency. Top-left: hackground image: top-right:- foreground object; bottom-left: scene composed with constant transparency; bottom-right: scene composed with gray-scale transparency, where the $\alpha$-map of the foreground object is a horizontal ramp starting with $m_{k}(0, y)=0$ on the left side and ending at $m_{k}(320, y)=25$ on the right side.

\subsubsection{SHAPE Representation}

The 2D shape of an object is defined by means of an $\alpha$-map $M_{k}$ of size $X Y$ pels:

$$
M_{k}=\left\{m_{k}(x, y) \mid 0 \leq x \leq X, 0 \leq y \leq Y\right\}, \quad 0 \leq m_{k} \leq 255
$$

The shape $M_{k}$ defines for each pel $\mathbf{x}=(x, y)^{T}$ whether it belongs to the object $\left(m_{k}(\mathbf{x})>0\right)$ or not $\left(m_{k}(\mathbf{x})=0\right)$. For an opaque object, the corresponding $\alpha$-values are 255, and for transparent object they range from 1 to 255 . Usually, the $\alpha$-map has the same spatial and temporal resolution as the luminance signal of the video sequence. In video-editing applications, the $\alpha$-map is used to describe object shape and object transparency. Let us assume that we have a background image $s_{b}(\mathbf{x})$, the object represented by image $s_{0}(\mathbf{x})$, and the $\alpha$-map $M_{0}(\mathbf{x})$. Overlaying the object on the backgroun is done according to

$$
s(\mathbf{x})=\left(1-\frac{M_{\mathrm{o}}(\mathbf{x})}{255}\right) s_{\mathrm{b}}(\mathbf{x})+\frac{M_{\mathrm{o}}(\mathbf{x})}{255} s_{\mathrm{o}}(\mathbf{x})
$$

As shown in Figure 12.3, the amplitude of the $\alpha$-map determines how visible the object becomes. We will describe the coding of binary object shapes, i.e., $m_{k}(\mathbf{x}) \in\{0,255\}$ in the next subsection, ollowed by description of the coding of gray-scale shape.

12.2.2 Binary Shape Coding

The MPEG-4 shape coder is known as the context-based arithmetic encoder (CAE) [2,3,24]. It work on macroblocks of size $16 \times 16$ pels that the MPEG-4 video encoder defines for a video object. In the ollowing paragraphs, shape encoding in ihra-nodo is deschber. Then, this a vishe is extend to include an inter-mode. The evaluation criteria for lossy binary shape coding are presented last.

\subsubsection{Intra-Mode}

The CAE codes pelwise information only for boundary blocks exploiting the spatial redundancy of the binary shape information to be coded. Pels are coded in scan-line order and row by row. In intra-mode, three different types of macroblocks are distinguished: transparent and opaque blocks are signaled as macroblock type. The macroblocks on the object boundary containing transparent as well as opaque pels belong to the third type. For these boundary macroblocks, a template of 10 pels is used to define the causal context for predicting the shape value of the current pel as shown in Figure 12.4a. For encoding the state transition, a context-based arithmetic encoder is used. The probability table of the arithmetic encoder for the 1024 contexts was derived from several sequences. With 2 bytes allocated to describe the symbol probability for each context the table size is 2048 bytes.

The template extends up to 2 pels to the left, to the right, and to the top of the pel to be coded. Hence, for encoding the pels in the two top and left rows of a macroblock, parts of the template are defined by the shape information of the already transmitted macroblocks on the top and on the left

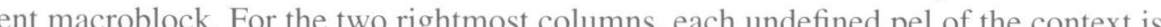
set to the value of its closest neighbor inside the macroblock.

In order to increase coding efficiency as well as to allow lossy shape coding, a macroblock may be subsampled by a factor of 2 or 4 resulting in a subblock of size $8 \times 8$ pels or $4 \times 4$ pels, respectively. The subblock is encoded using the encoder as described above. The encoder transmits to the decoder the subsampling factor such that the decoder decodes the shape data and then up-samples the decoded subblock to the original macroblock size. Obviously encoding the shape using a high subsampling factor is more efficient but the decoded shape after up-sampling may or may not be the same as the original shape. Hence, this subsampling is mostly used for lossy shape coding.

Depending on the up-sampling filter, the decoded shape can look somewhat blocky. During the MPEG evaluation process, two filters have been found to perform very well: a simple pel replication filter combined with a $3 \times 3$ median filter and slightly better performing adaptive nonlinear upsampling filter. MPEG-4 decided to adopt the adaptive filter with a context as shown in Figure 12.5 [21]. The value of an up-sampled pel is determined by thresholding a weighted sum of the pels of its

The efficiency of the shape coder differs depending on the orientation of the shape data. Therefore, the encoder can choose to code the block as described above or transpose the macroblock prior to rithmetic coding.

Within the MPEG-4 standardization process, the quality of shape coding was controlled by a threshold, AlphaTH, which defines the maximum number of erroneously coded pels in a macroblock without considering effects like change in connectivity, impact on overall object shape, temporal shape

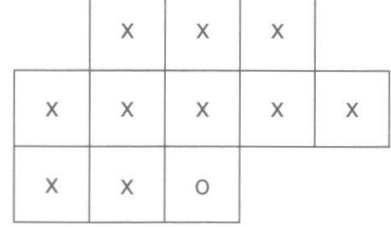

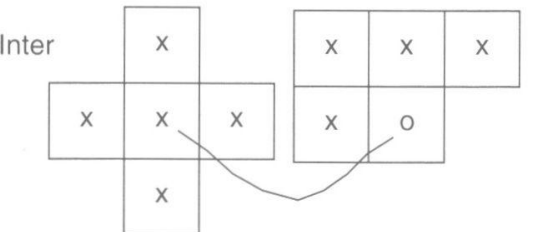

FIGURE 12.4 Templates for defining the context of the pel to be coded (o), where (a) defines the intra-mode context and (b) defines the inter-mode context. The alignment is done after motion compensating the 


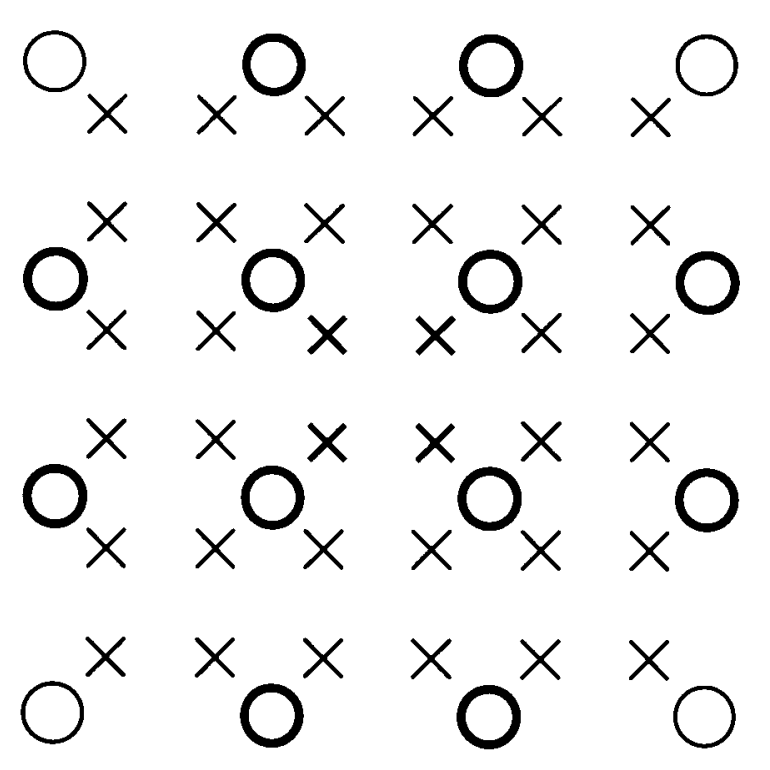

FIGURE 12.5 The up-sampled pels $(x)$ lie between the locations of the transmitted pels $(0)$ from the subsample macroblock shape information. Neighboring pels (bold o) defining the values of the pels to be up-sample

variation or the look of the object including its texture. Therefore, lossy shape coding achieves better results if these effects are considered.

\subsubsection{Inter-Mode}

In order to exploit temporal redundancy in the shape information, the coder described above is extended by an inter-mode requiring motion compensation and a different template for defining the context.

For motion compensation, a 2D integer pel motion vector is estimated using full search for each macroblock in order to minimize the prediction error between the previous coded VOP shape $M_{k-1}^{\prime}$ and the current shape $M_{k}$. The shape motion vectors are predictively encoded with respect to the shape motion vectors of neighboring macroblocks. If no shape motion vector is available, texture a new template designed for coding shape in inter-mode as shown in Figure 12.4b.

The template defines a context of 9 pels resulting in 512 contexts. The probability for one symbol is described by 2 bytes giving a probability table size of 1024 bytes. Four pels of the con are neighbors of the pel to be coded, 5 pels are located at the motion-compensted the contex the previous VOP. Assuming that the motion vector $\left(d_{k} d_{j}\right)^{T}$ points from the current $V O P_{k}$ to previous coded $V O P^{\prime}$, the part of the template located in the previ fold

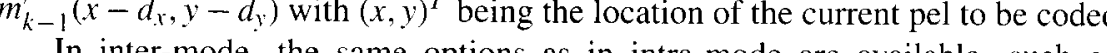

In inter-node, the same options as in intra-mode are available, such as subsampling and achieved by simply carrying out motion compensation is sufficient thus saving bits by aventation code the prediction error. The encoder can select one of the seven modes for the by avoiding to tion of each macroblock: trensprent opelo and inter with or without shape motion vectors and predicion error coling. These diffect oplons with optional subsampling and transposition allow for encoder implementations of different coding efficiency and implementation complexity.

\subsubsection{Evaluation Criteria for Coding Efficiency}

In order to compare the performance of different shape coders, evaluation criteria have to be defined. Within MPEG-4, there are two quality measures for objectively assessing the quality of coded shape parameters. One is the maximum of the minimal Euclidean distance $d_{\max }^{*}$ (peak deviation) between each coded contour point and the closest contour point on the original contour. This measure allows for an easy interpretation of the shape quality. However, if lossy shape coding results in changing the topology of an object due to opening, closing, or connecting holes, the peak deviation $d_{\max }^{*}$ is not a useful measure. Therefore, a second measure $d_{n}$ was used, which is the number of erroneously represented pels of the coded shape divided by the total number of pels belonging to the original shape. Since different objects can have very diferent ratios of contour pets to interior pels, a given value for $d_{n}$ only allows to compare with other $d_{n}$ of different approximations of the same video object. $d_{n}$ by itself does not provide sufficient information about the shape quality. Hence, some evaluations are done just providing the number of erroneously coded pels.

It was found that the objective measures truthfully reflect subjective quality when comparing different bitmap-based shape coders or when comparing different contour-based shape coders. For lossy shape coding, the bitmap-based shape coders create blocky object shapes whereas contourbased shape coders create an object shape showing polygon edges. Since the two classes of shape coders give different results, a comparison between these two classes has to be done subjectively.

\subsubsection{Gray-Scale Shape Coding}

Gray-scale $\alpha$-maps allow 8 bits for each luminance pel to define the transparency of that pel. As shown in Figure 12.3, transparency is an important tool for composing objects into scenes and special effects. Two types of transparencies are distinguished: binary $\alpha$-maps with objects of constant transparency and arbitrary $\alpha$-maps for objects with varying transparency.

\subsubsection{Objects with Constant Transparency}

For a transparent object that does not have a varying transparency, the shape is encoded using the binary shape coder and the 8 -bit value of the $\alpha$-map. In order to avoid allasing, gray-scale $\alpha$-maps usually have lower transparency values at the boundary. Blending the $\alpha$-map near the object boundary can be supported by transmitting the coefficients of a $3 \times 3$ pel FIR filter that is applied to the $\alpha$-map within a stripe on the inner object boundary. The stripe can be up to 3 pels wide.

\subsubsection{Objects with Arbitrary Transparency}

For arbitrary $\alpha$-maps, shape coding is done in two steps [5,7]. In the first step, the outline of the object is encoded as a binary shape. In the second step, the actual $\alpha$-map is treated like the luminance of an object with binary shape and coded using the MPEG-4 texture coding tools: motion compensation, DCT, and padding. The passing extrapolates the object texture for the background pels of a boundary block.

\subsubsection{TeXtuRe CODING OF BOUNDARY Block}

For motion-compensated prediction of the texture of the current VOP, the reference VOP is motioncompensated using block motion compensation. In order to guarantee that every pel of the current 
VOP has a value to be predicted from, some or all of the boundary and transparent blocks of the reference VOP have to be padded. Boundary blocks are padded using repetitive padding as follow First, boundary pels are replicated in the horizontal direction, and then they are replicated in the vertical direction. If a value can be assigned to a pel by both padding directions, then an average value is assigned to that pel. Since this repetitive padding puts a significant computational burden on the decoder, a simpler mean padding is used in the second step. Transparent macroblocks bordering boundary blocks are assigned to an average value determined by the pels of its neighboring padded blocks.

In order to encode the texture of a boundary block, MPEG-4 treats the macroblock as a regular macroblock and encodes each block using an $8 \times 8$ DCT. The texture is decoded using convention processing, then discards all information that fats outside of the decoded shape. In order to increa coding efficiency, the encoder can choose the texture of pels outside of the object such that the bit rat is minimized. This non-normative process is also called padding [25]. For intra-mode, a low-pas extrapolation titer was developed, while for inter-mode, setting these pels to 0 was found to perform well in terms of coding efficiency.

\subsubsection{VIDEO CODER ARCHITECTURE}

Figure 12.6a shows the block diagram of an object-based video coder [35]. In contrast to the block diagram shown in the MPEG-4 standard, this diagram focuses on the object-based mode in order to allow a better understanding of how shape coding influences the encoder and decoder. Image analysis creates the bounding box for the current VOP $s_{k}$ and estimates texture and shape motion of the current $\mathrm{OP}$ with respect to the reference $\mathrm{VOP} s_{k-1}$. Shape motion vectors of transparent macroblocks are set to 0 . Parameter coding encodes the parameters predictively. The parameters get transmitted and decoded, and the new reference VOP is stored in the VOP memory and also handed to the composito of the decoder for display.

The increased complexity due to the coding of arbitrarily shaped video objects becomes evident in Figure 12.6b, which shows a detailed view of the parameter coding. The parameter coder firs encodes the shape of the boundary blocks using shape and texture motion vectors for prediction. Then, shape motion vectors are coded. The shape motion coder knows which motion vectors to code by analyzing the possibly lossily encoded shape parameters. For texture prediction, the reference VOP is padded as described above. The prediction error is then padded using the original shape parameters to determine the area to be padded. Using the original shape as a reference for padding is again an encoder choice not implemented in the MPEG-4 Reference Software [20] (MoMuSy ersion). Finally, the texture of each macroblock is encoded using DC

\subsection{CODEC OPTIMIZATION}

The shape coding tools described in the previous subsection provide the basic techniques that are used to efficiently represent shape data. In order to use these tools most effectively, optimizations arious points in the encoder and decoder must be considered. Several key issues are outlined below nd discussed further in this section.

Prior to encoding, preprocessing of shape data may be helpful to simplify the information to be coded, while still providing an accurate representation of the object. During the encoding, rate control is needed at the encoder to allocate bits to achieve a maximum quality subject to rate an buffer constraints. Rate-distortion $(R-D)$ models of the shape (and texture) data may be used to atleviate some of the computations involved in making optimal coding decisions. Furthermore, error control may be used to minimize data loss incurred during transmission and error propagation in the

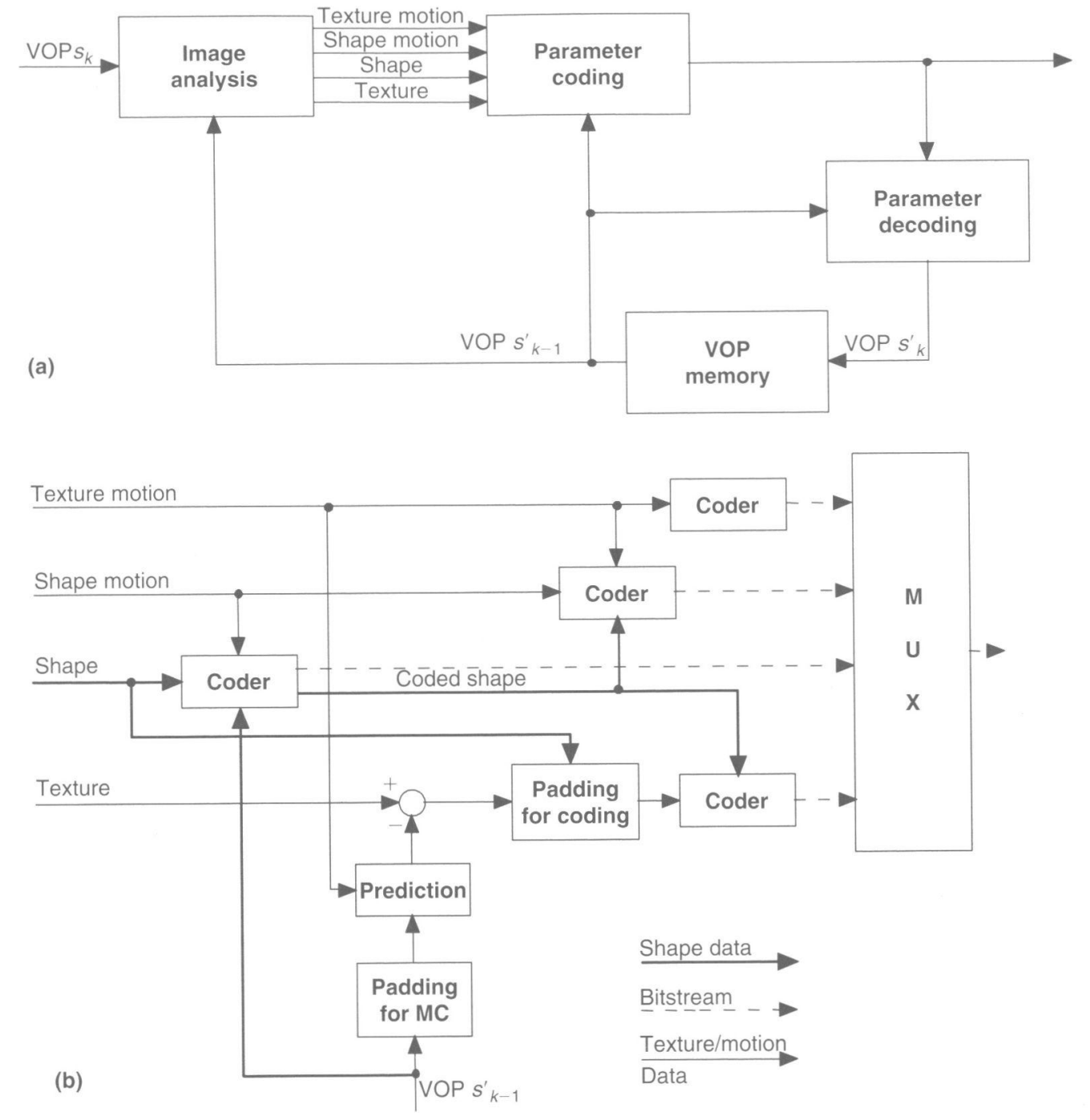

FGURE 12.6 Block diagram of the video encoder (a) and the parameter coder (b) for coding of arbitrarily shaped video objects.

In addition to the the above encoder issues, postprocessing techniques after decoding also play an important role in reconstructing the object and scene data, and the scene. For one, if errors during applied. Also, given multiple objects in a scene, composition of these objects is also required.

\subsubsection{PREPROCESSING}

The decoder has no possibility to find out whether the encoder uses lossless or lossy shape coding and what shape coding strategy the encoder uses or padding algorithm for coding is used. In its reference implementation of a video coder. MPEG-4 chose to control lossy shape coding by using an AlphaTH hreshold. This threshold defines the maximum number of incorrectly coded pels within a boundary 
block and allows the topology of the shape to change. Often, isolated pels at the object boundary are odet as ant of the object. Using morphogical fiters to smooth object houndaries provide a much more predictable quality of a lossily encoded shape [35].

12.3.2 Rate-Distortion Models

For texture coding, a variety of models have been developed that provide a relation between the rate

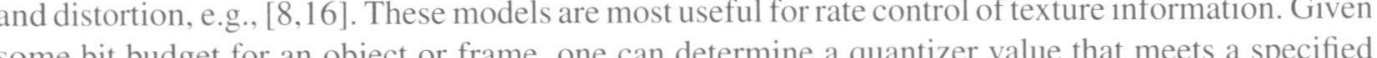
on encoded in an effort to optimize the coding efficiency in a computationally efficient way.

In the case of shape coding, analogous models have been developed, mainly for use in the alyis tage of an object-based encoder. The primary motivation to develop such models is to avoid performing actual coding operations to obtain the $R-D$ characteristics of the binary shape. In the case of MPEG-4 shape coding, this involves down-sampling and arithmetic coding operations to obtain the rate, and up-sampling and difference calculations to compute the distortion at various scales. Figure 127 shows sample images for binary shapes at different resolutions and after upsampling. Accurate estimates of the $R-D$ characteristics can play a key role in optimizing the bit
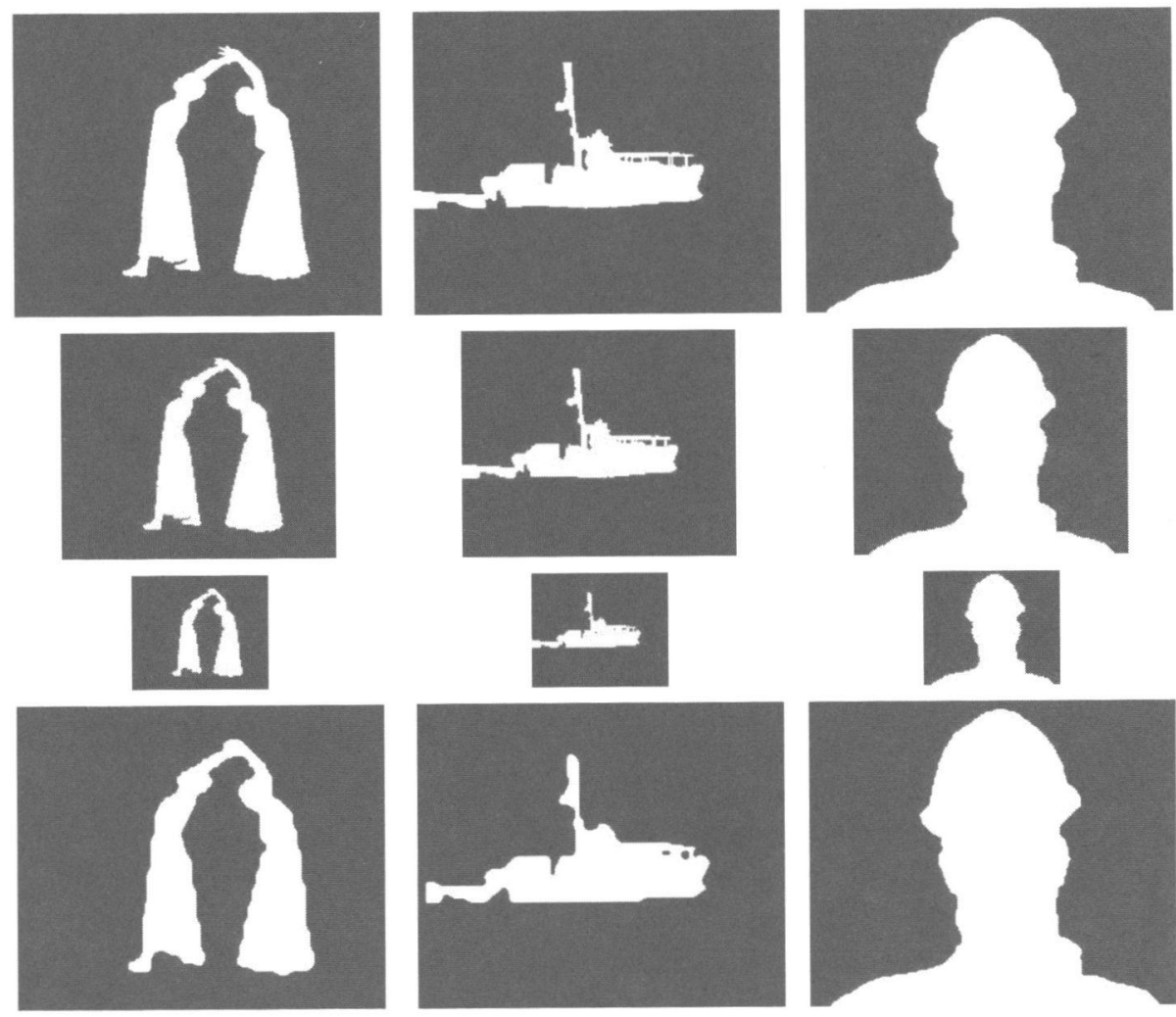

FIGURE 12.7 Sample test shapes from the Dancer. Coastguard, and Foreman sequences. The first row shows the original shapes at full resolution. The second and third rows show the shapes down-sampled by factors 2 and 4 according to the MPEG-4 standard. The bottom row shows the up-sampled reconstructed shapes from (Dancer and Coastguard) and half-scale image (Foreman) according to the MPEG-4 standard. allocation for the binary shape among blocks and between shape and texture coding with much lower complexity. Also, knowing the expected rate for shape can stabilize the buffer occupancy of a video coder, especially for low bit-rate video coding [50]

In the following, we review several approaches that have been proposed for modeling the $R-D$ characteristics of binary shape and discuss the performance of each. Throughout this section, we aim to model the characteristics of the MPEG-4 CAE-based shape coder described in Section 12.2. Also we assume shapes are coded in intra-mode only, unless otherwise specified.

or the purpose of this subsection, the modeling problem is formally stated as follows. Let $(R, D)$ denote the $R-D$ values for a binary shape that is coded at resolution $k$. Using input parameters, $\theta_{1}$ which represent features extracted from the shape data, and modeling function, $f(\cdot)$, we consider the approximation,

$$
(R, D)_{k} \approx f\left(\theta_{i}\right)
$$

The first attempt to solve this problem attempted to categorize all possible binary patterns over $2 \times 2$ neighborhood into $N$ states [52]. This approach was referred to as state partitioning. As expected the rate is predicted well by this approach, but the distortion suffers from large prediction error. The reason for this is because the rate could be acetratery modeled from a fixed $2 \times 2$ neighborhood, such that the 10-bit states used by CAE can be correctly collapsed into one of the avaliable states. The distortion, on the other hand, is not modeled so aceurately because the actual up-sampling proces uses a 12-pet neighborhood and estimating distortion based on the $2 \times 2$ pixels is not sufficient.

An improved probabilistic approach for modeling the $R-D$ characteristics of binary shape was proposed in [53]. In this work, the shape model is based on the statistics (or moments) that one can extract fom has intes resemble the type of data that we are trying to code. At the same time, this model should be able to make distinctions between differe same she differen

In [10], a Markov random field (MRF) model that is able to represent the fine structures of an image is presented. The model relies on three parameters: edge, line, and noise. It has been show in [53] that the moments of this mode, which are considered sufficient statistics of the distribution, exhibit favorable properties for modeling as outhined above.

To estimate the rate and distortion a various seates, a simple muttilayer feed-forward network has been proposed in [53], where the input to this network are the statistical moments of the MR model that are calculated from the original shape image, and the output is the estimated rate an distortion of the shape data at different scales.

The plots in Figure 12.8 provide a comparison of the actual rates and output rates of the neura network for each or the three scales. In these plots, the first 100 frames correspond to the training set and the fext 75 frames correspond to the testing see. We can see that that the neural network does indeed provide close estimates to the actual rate and follows the trends quite weth for both the training and testing data. Similaty, the plots in Figure 12.9 provide a comparison of the actual distortions and

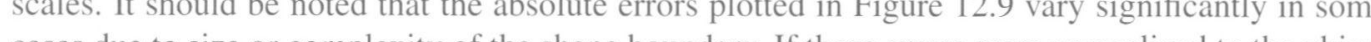
cases due to size or complexity of the shape boundary. If these errors were normalized to the object size, the relative entors would be mich closer.

White the resaltat MRF parameters can be used to model multiscale rate-distortion characteristics or binary shape, and do so with information provided at the full resolution has been proposed based ophe the linear model is applied to both intra- and inter-coded shape. 


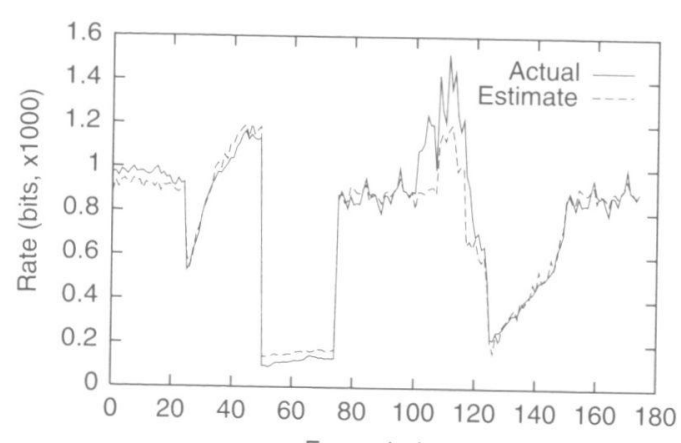

Frame index

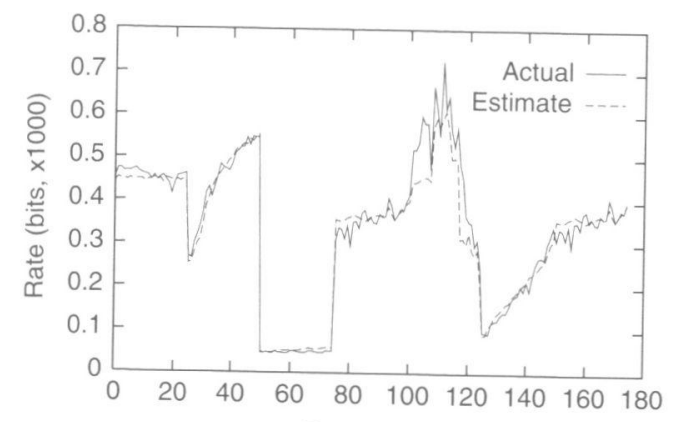

Frame index

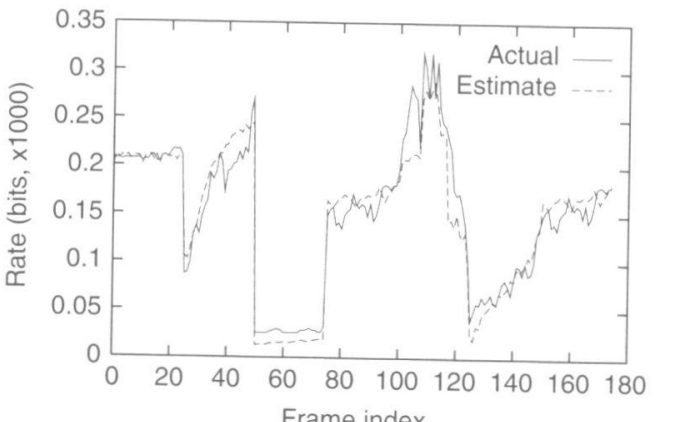

Frame index

HGURE 12.8 Comparison of actual rates and output rates of neural network from [53] at fult-sate (top). half-scale (bottom-left), and quarter-scale (bottom-right). The first four clips (corresponding to the first 100 raames) are part of the training set, while the following three clips (corresponding to the remaining 75 frames) are part of the test set.
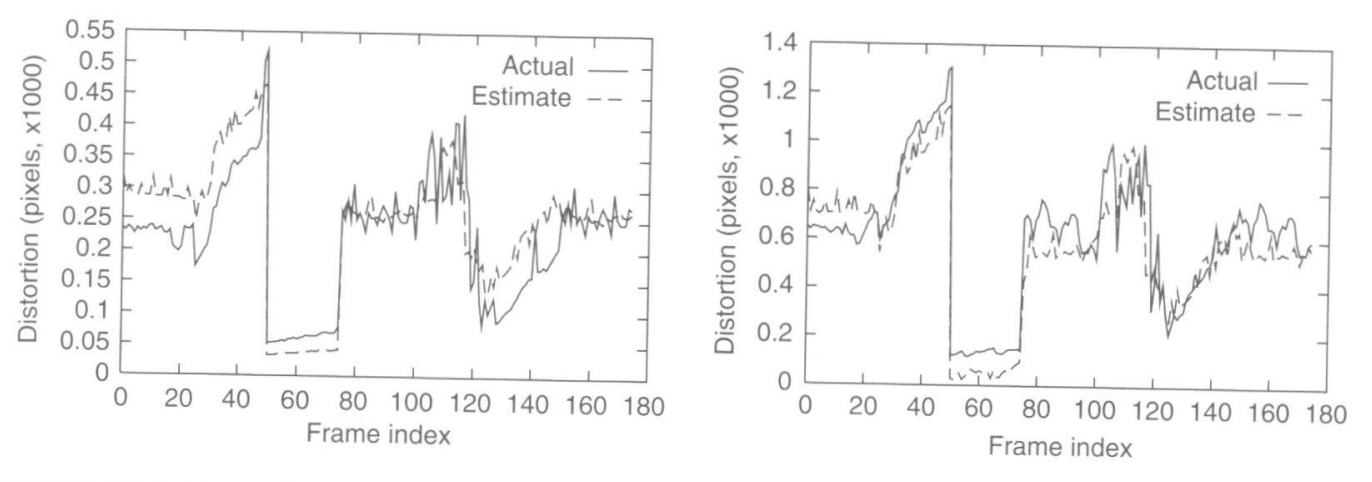

FIGURE 12.9 Comparison of actual distortions and output distortions of neural network from [53]. Distortion is measured between original and reconstructed binary map from half-scale (left) and quarter-scale (righ)-The first four clips (corresponding to the first 100 frames) are part of the training set, while the following three clips (corresponding to the remaining 75 frames) are part of the test set.

As stated in [6], the rate model is given by

$$
\tilde{R}_{i}=a_{i} n+b_{i}
$$

where $n$ is the number of boundary blocks, $a_{i}$ and $b_{i}$ are model parameters, and $i$ denotes the resolution scale. Linear regression is used to estimate the model parameters based on past data points.
Similar to the rate model, the distortion model is given by

$$
\tilde{D}_{i}=c_{i} n+d_{i}
$$

where $c_{i}$ and $d_{i}$ are model parameters, which are also calculated using linear regression on past data points. However, it has been observed that more complex boundaries will always produce more distortion, therefore a shape complexity measure based on a normalized perimeter has been introduced:

$$
\kappa=\frac{\sum_{l=1}^{n} p_{l}}{S}
$$

where $p_{l}$ denotes the perimeter of each boundary block and $S$ the number of nontransparent pixels in the shape. This complexity measure is factored into the distortion model in a multiplicative way such that the new distortion model become

$$
\tilde{D}_{i}=c_{i} \kappa n+d_{i}
$$

Simulation results in [6] confirm the accuracy of the above approach for both intra- and inter-coded shapes at various levels of resolution.

\subsubsection{Rate Control}

In object-based coding, the problem of coding additional shape information becomes critical at lower bit rates. At higher bit rates, the shape bits occupy a much smaller percentage of the overall rate. In the following, we first describe a buffering policy to account for the additional shape information in object-based coding. With object-based coding, there is also some flexibility in allocating rate among texture and shape information, so we also discuss bit allocation among texture and shape. In both cases, the bits used for shape are estimated from the $R-D$ models described in Section 12.3.2. Finally, we discuss how rate control decisions for different objects may impact the overall composition of a scene.

\subsubsection{Buffering Policy}

In frame-based rate control, the bits used to encode a frame, $T_{c}$, are added to the buffer. Let the buffer occupancy at time $t$ be denoted by $B(t)$. To help ensure that the buffer will not overflow, a frameskip parameter, $N$, is set to zero and incremented until the following buffer condition is satisfied:

$$
B(t)<\gamma B_{s}
$$

where $B_{\mathrm{s}}$ is the size of the buffer, and the value of $\gamma$ denotes a buffer margin having a typical value of 0.8 . In the above, the updated buffer level is given by

$$
B(t)=B(t-\tau)+T_{\mathrm{c}}-T_{\mathrm{d}}(N+1)
$$

where $B(t-\tau)$ denotes the buffer level of the previously coded frame and $T_{\mathrm{d}}$ is the amount of bits drained from the buffer at each coding time instant.

In object-based coding, the buffering policy must account for the high percentage of shape bits at low bit rates. Let the estimated number of bits to code the shape for all objects in the next frame be denoted by $T_{\text {shape }}$ Then, to ensure that there are a sufficient number of bits to code the texture in the next frame, the buffer condition given by Eq. (12.8) is slightly modified as

$$
B(t)+T_{\text {shape }}<\gamma B_{\mathrm{S}}
$$


Another way to view the modified buffer condition is that the buffer margin is adaptively lowered according to the estimated hits for shape. In this way. if a relatively large number of bits are estimated for the shape, the rate control can account for these additional bits by skipping frames and allowing for bill allow a sufficient number of bits to code all the information in the nex frame. without compromising the spatial quality more than necessary.

\subsubsection{Bit Allocation}

Generally speaking. bit allocation aims to minimize distortion subject to rate and buffer constraints. In an object-based coding framework. allocation of bits used for both shape ind texture data could be performed in a joint manner. However. since the distortion metrics for shape and texture are differen, it is not straightforward to derive a single cost function that accounts for both metrics. It should be noted that binary shape distortion is usually defined as the ratio of error pixels to the total number of nontransparent pixels. while texture distortion is defined according to mean-squared error between original and reconstructed pixel values.

Let the total rate budget for an object be given by $R=R_{\mathrm{t}}+R_{\mathrm{w}}$, where $R_{\mathrm{t}}$ are the bits allocated for texture (including motion) and $R_{\mathrm{s}}$ are the bits allocated for shape. Given this allocation, the optimal texture and shape coding modes for each block in the object could be determined separately using. or example, conventional Lagrangian multiplier techniques. If $R-D$ models are used to estimate the operating points for texture and shape at various quantizer values and conversion ratios, respectively,
then some of the computational burden could be alleviated. The problem is then reduced to one of then some of the computational burden could be alleviated. The problem is then reduced to one of allocating the total bits for an object among $R_{\mathrm{t}}$ and $R_{\mathrm{s}}$. This problem may also be posed at the frame vel in which the optimization is conducted for all objects in a scene.

The main difficulty with the above approach is allocating bits between shape and texture. To overcome this problem, a practical approach has been proposed in [50], where rather than allocating a specific number of bits to shape, the maximum distortion for shape is controlled through the AlphaTH threshold instead. In this way, the shape will consume some portion of the total rate budget for a frame subject to a maximum distortion constraint and the remaining bits would be allocated to the texture. In [50]. a method to dynamically adapt the AlphaTH threshold according to the buffer
fullness and quality of the texture encoding has been proposed.

\subsubsection{ERror Control}

It is largely recognized that intra-refresh can play a major role in improving error resilience in video coding systems. This technique effectively minimizes error propagation by removing the temporal relation between frames. Although the intra-refresh process decreases the coding efficiency, it will significantly improve error resilience at the decoder, which increases the overall subjective impact in the presence of errors in the transmission.

A key aspect to consider in applying intra-refresh schemes is to determine which components of the video to refresh and when. For object-based video, both shape and texture data must be considered. In [45], shape refreshment need (SRN) and texture refreshment need (TRN) metrics have been proposed. The SRN is defined as a product of the shape error vulnerability and the shape concealment difficulty, while the macroblock-based TRN is similarly defined as a product of the texture error vulnerability and the texture concealment difficulty. In both cases. the error vulnerability measures the statistical exposure of the shape/texture data to channel errors and the concealment difficulty expresses how difficult the corrupted shape/texture data is to recover when both spatial and temporal error concealment techniques are considered.

Based on the above refreshment need metrics, novel shape and texture intra-refreshment schemes have been proposed in [46]. These schemes allow an encoder to adaptively determine when the shape and texture of the various video objects in a scene should be refreshed in order to maximize the decoded video quality for a certain total bit rate.

\subsubsection{Post-Processing}

Two specific needs for postprocessing shape data are considered in this subsection. First. given that the shape data defines objects in a scene, composition and alpha-blending techniques are required to generate a complete scene consisting of multiple objects. Second, considering that errors may occu

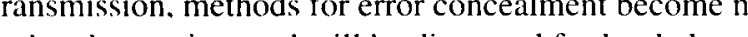
performed at the receiver and will be discussed further below.

\subsubsection{Composition and Alpha Blending} As mentioned earlier, composition issues may arise due to lossy coded shape or the coding of multiple
objects at different temporal rates. In [50], undefined pixels due to lossy shape coding were simply assigned a gray value. Since maximum distortion in a block was controlled using AlphaTH, which assigned a gray value. Since maximum distortion in a block was controlled using Alpha H. Which
only took values in the range [0.64]. the impact on visual quality was not noticeable. With higher
levels of shape distortion, more sophisticated methods would be required to recover the pixel values for the texture. In [29], object coding with different temporal rates was considered. In this work undefined pixels were assigned values from the reconstructed object with minimum distance to the undefined pixel coordinate.

\subsubsection{Frror Concealment}

Concealment techniques could be divided into two categories: temporal and spatial. Temporal concealment techniques rely on shape information from previous time instants to do the concealmen while spatial concealment techniques use information only from the current time instunt. Several techniques for spatial and temporal concealment are outlined below.

The earliest known attempt to address this concealment problem for shape was proposed in [13]. where the idea of extending conventional motion-compensated concealnint techniques for texture to shape was explored. In this way, when a given block of shape data is corrupted, the decoder conceals it by copying a block of shape data from the previous time instant. This could be achieved by simply copying the co-located block of shape data from the previous time instant. Alternatively. a motion vector estimate may be obtained such that the corrupted block in the current time instant is replaced by a displaced shape block from the previous time instant. Temporal concealment was further investigated in $[40]$ considering that the boundary of the shape data of a given video object does not change significantly in time and thus these changes can be described by a global motion model. Based on this assumption, the global motion parameters are estimated at the encoder and sen along with the encoded bitstream to the decoder. With this method, the decoder would apply globa motion compensation using parameters derived at the encoder to restore the corrupted contour, the fill in the concealed contour to recover the entire shape. An extension of this approach was proposed in [48], which eliminates the need for an encoder to send additional information by performing the estimation of global motion parameters at the decoder using available shape data. Additionally, this approach improves performance by adding a motion refinement step to better deal with shapes that have some local motion

The above temporal coust information that can significantly enhance the recovery of shape data. especially in cases where the shap does not change much. However, when the shape changes significantly over time or concealment is applied to still or intra-coded shapes. spatial concealment techniques should be used instead.

Spatial shape concealment was first addressed in [43]. In this work, the shape was modeled with a binary MRF and concealed based on maximum a posteriori (MAP) estimation. According to this algorithm, each missing shape element in a missing block is estimated as a weighted median of the neighboring shape elements that have been correctly decoded or concealed. where the weights ar assigned adaptively based on the likelihood of an edge in that direction. For each missing shape 


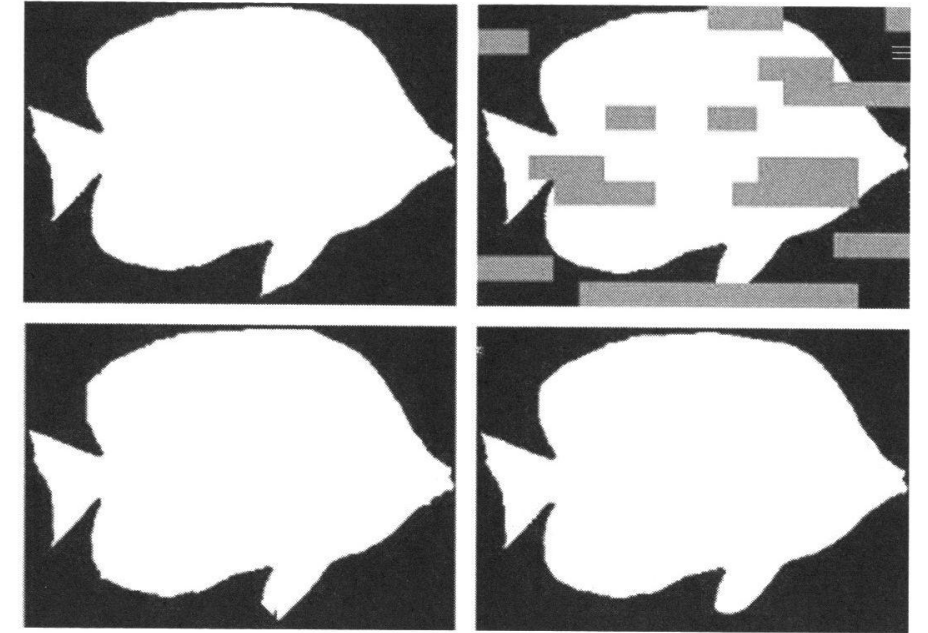

FIGURE 12.10 Performance comparison of error concealment techniques. Top-left: original shape image; top-right: corrupted shape image with $25 \%$ of total blocks lost; bottom-left: shape image recovered using method of Shirani et al. [43]; bottom-left: shape image recovered using method of Soares and Pereira [47].

block, this procedure is iteratively repeated until the algorithm converges. Additionally, if several consecutive missing blocks have to be concealed, the procedure is recursively applied to all the missing shape blocks. This medhod obviously has some computational burdens, but may also suffer in concealing isolated blocks since only local statistics of the data are considered. In an attempt to surrou the available sure are fully denche the Figure 12.10 con thas ens that of $S$. ha pere por

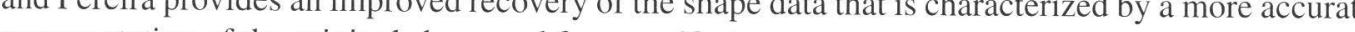
representation of the oricinal shape and fewer atifacts.

\subsection{APPLICATIONS}

This section describes how shape coding and object-based video coding, in general, could be applied for surveillance and interactive TV applications.

\subsubsection{Surveillance}

Here we describe a surveillance application system that utilizes object-based coding techniques to achieve efficient storage of video content [49]. In this system, certain inaccuracies in the reconstructed scene can be tolerated, however, subjective quality and senanics of the scene must be strictly maintained. As shown in Figure 12.11, the target of this system is the long-term archiving of survelllance video, where several months of video content from multiple cameras would need to be stored.

One of the major advantages of object-based coding is that each object can vary in its temporal quality. However, in terms of rate-distortion metrics, only minimal gains in coding efficiency could be achieved [51] since the composition problem typically limits the amount of temporal frame skipping

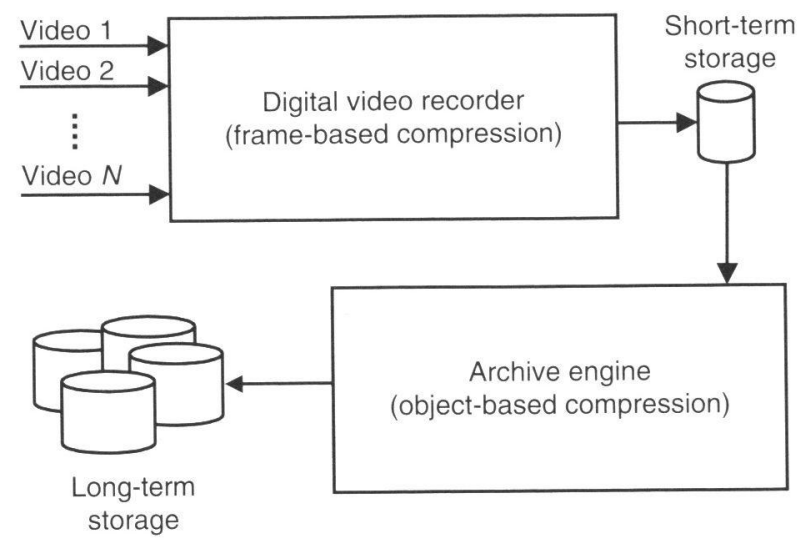

HCURE 1211 Application system for surveillance emploving object-based coding for long-term archive of video content.

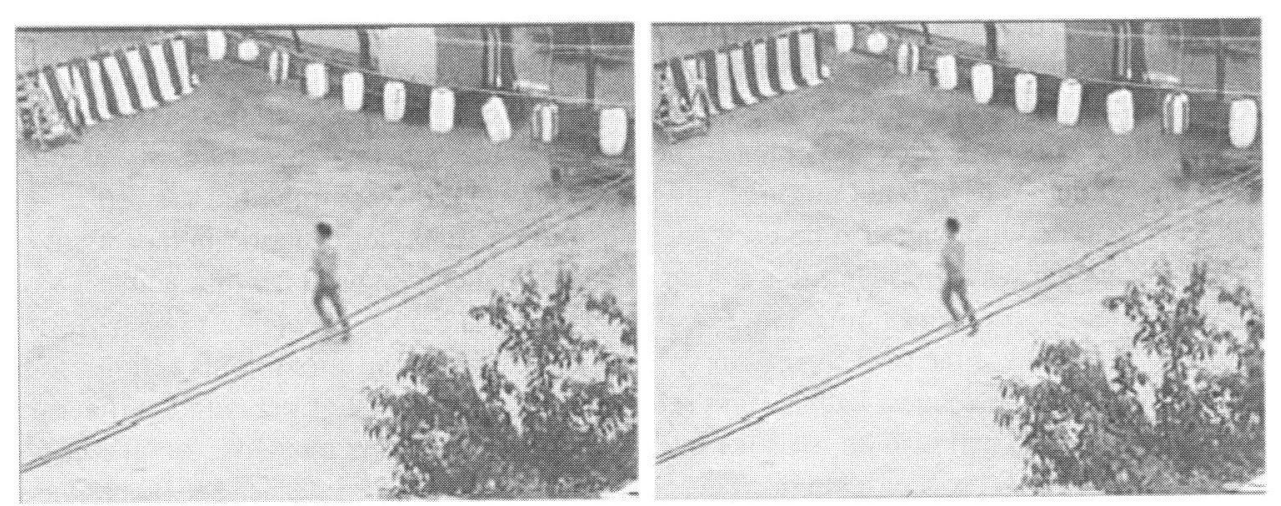

FIGURE 12.12 Sample reconstructed frame of festA sequence. Left: frame-based reconstruction; right object-based reconstruction.

of the background object. Fortunately, in the surveillance system being discussed here, obtaining the full background without any foreground objects is not a problem. Also, subjective video quality i the main consideration

In this system, a single background image is compressed using frame-based coding, and the sequence of segmented foreground objects are compressed using object-based coding; the backgroun image is simply repeated for each reconstructed frame. Performing the object-based compression in this way gives rise to differences in the background pixels, especially for outdoor scenes that, for example, have swaying trees and objects due to wind conditions.

To demonstrate the effectiveness of this approach, the above object-based coding methodology is applied to several surveillance test sequences. Sample reconstructed frames of one sequence using frame- and object-based coding are shown in Figure 12.12. In this test sequence, wind is blowing the striped curtain, tree branches, and hanging ornaments, all of which are coded and accurately represented by the frame-based coding result. However, for the object-based coding result, these moving background elements are not tecorded and fy he noving forctround objectiscoded at each time instant. Semantically, however, these sample frames are equivalent. Table 12.1 summarizes the 


\begin{tabular}{|c|c|c|c|c|c|c|}
\hline \multicolumn{7}{|c|}{$\begin{array}{l}\text { TABLE } 12.1 \\
\text { Comparison of Storage Requirements (in KB) } \\
\text { for Frame-Based and Object-Based Coding }\end{array}$} \\
\hline Sequence & fest A & festB & festc & festD & rain & Iree \\
\hline Fram & 178 & 173 & 35 & 116 & 78 & 43 \\
\hline Object-based & 17 & 18 & 13 & 18 & 22 & 10 \\
\hline \% Savings & 90.5 & 89.6 & 62.9 & 84.5 & 71.8 & 76.7 \\
\hline
\end{tabular}

storage requirements over a broader range of sequences; it is clear that object-based coding provides favorable savings in the bits required to store the compressed video sequences.

\subsubsection{InTERACTIVE TV}

With the increasing demand for access to information for handicapped people, TV has to become accessible for the hearing impaired. Overlaying a person signing the spoken words over the video will enable this functionality. Transmitting this person using object-based coding and overlaying this object over the regular TV program enables the user to select whether this optional video object should be presented in order to not diminish the presentation for the nonimpaired viewers.

MPEG-4 shape coding can also be used for transmitting shape information independent of the video signal. This allows for transmitting a map of labels that can be used for interactive TV. If the user moves a cursor with the remote control over the video and clicks, the action to be performed is determined by the label of the map corresponding to the position of the cursor. This action can sele-t iems for teleshopping or request background information on the object or person identified

Object-based video can also be used for overlaying news reporters over live footage at the TV receiver instead of at the broadcast studio. This is of special interest for Internet TV, where the same news story might require reporters talking in different languages. In this scenario, the background video is sent as an rectangular-shaped video object on one channel and the news reporter with the desired language represented as an arbitrarily shaped video object is selected on the second channel.

Multipoin video conference systems would like to present the remote participants in an homogeneous environment to the local participant. This can be easily achieved when each participant is an a video objects on a common background and present the result to the local participant.

\subsection{CONCLUDING REMARKS}

This chapter has described the shape coding tools that have been adopted in the MPEG-4 coding standard. The context-based arithmetic encoder uses a template to define the context for coding the current pel of a binary shape bitmap. If objects have arbitrary transparency, then these values are coded using MPEG-4 tools like DCT and motion compensation. The integration of a shape coder with texture coding for object-based video coding requires use of texture extrapolation also known as padding. Lossy shape coding may create pels with undefined texture requiring the use of similar extrapolation techniques as well as concealment techniques. For video coding, binary shape coding

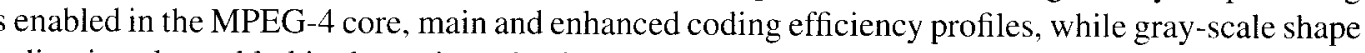
coding is only enabled in the main and enhanced coding efficiency profiles.
Beyond the fundamental shape coding techniques, we have discussed several issues related to encoder optimization, including $R-D$ modeling of binary shape, rate control, and error control. A linear $R-D$ model has shown to be effective in predicting rate and distortion of shape at various resolutions. Based on such models, we have reviewed buffering policies for maintaining a stable buffer control techniques that techniques to distribute the rate among shape and need metrics have been described.

Postprocessing of shape data has also been covered in this chapter. In particular, an overview of various error-concealment techniques for the recovery of lost data during transmission has been described. Temporal concealment techniques are advantageous since there is access to past information to improve recovery results; however, spatial concealment is still needed in cases where the shape changes significantly over time or for concealment of still or intra-coded images.

Finally, two promising applications of shape coding have been presented, including the use of object-based coding for long-term archiving of surveillance video resulting in bit savings between 60 and $90 \%$, and interactive televisio

\section{REFERENCES}

1. S.M. Aghito and S. Forchhammer, Context based coding of binary shapes by object boundary straightness analysis, IEEE Data Compression Conference, UT, USA, March 2004

N. B Cady and F Bosson, Shape compression of moving bje CCITT, Facsimile Coding Schemes and Coding Functions for Group 4 Facsimile Apparatus, CCIT Recommendation T.6, 1994

W. Chen and M. Lee, Alpha-channel compression in video coding, ICIP 97, Special session on shape coding, Santa Barbara, 1997

nodels for MPEG-4 shape coding, IEEE Trans. Circuits Syst. Video Technol., 14 (2004) 869-873.

T. Chen, C.T. Swain, and B.G. Haskell, Coding of subregions for content-based scalable video, IEEE Trans. Circults Syst. Wideo TeAm 7 (1997) 256-260.

The

Version $89 a$, CompuServe Incorporated, Columbus, OH, July 1990

10. X. Descombes, R.D. Morris, J. Zerubia, and M. Berthod, Estimation of Markov random field parameters using Markov chain Monte Carlo maximum likelihood, IEEE Trans. Image Process., 8 (1999)954-962

1. J.-C. Duford, BIFS: scene description, in The MPEG-4 Book, T. Ebrahimi and F. Pereira, Eds., IMSC Press Multimedia Series, Prentice-Hall, Upper Saddle River, 2002, pp. 103-147.

2. M. Eden and M. Kocher, On the performance of a contour coding algorithm in the context of image coding. Part M. Cos Kur segment coding, Signal Pabess.8 (1985) 381-386.

3. M.R. Frater, W.S. Lee, M. Pickering, and J.F. Arnold, Error concealment of arbitrarily shaped video Chets, Preedings

4. H. Freeman, On the encoding of arbitrary geometric configurations, IRE Trans. Electron. Comput, P. Gerken, Object-based analysis-synthesis coding of image sequences at very low bit rates, IEEE Trans. Circuits Syst. Video Technol, 4 (1994) 228-235.

6. H.M. Hang and J.J Chen, Source model for transform video coder and its application - Part fundamental theory, IEEE Trans. Circuits Syst. Video Technol., 7 (1997) 287-298. 
17. M. Hoetter, Optimization and efficiency of an object-oriented analysis-synthesis coder. IEEE TransCircuits Syst. Video Technol., 4 (1994) 181-194. 8. M. Hötter. Object-oriented analysis-synthesis

Process.: Image Commun., 2 (1990) 409-428.

19. ISO/EC JTC1/SC29/WG1, IS11544 - Coded Representation of Picture and Audio Information Progressive Bi-Level Image Compression. ISO. 1992.

20. ISO/IEC, JTC1/SC29/WG11. IS14496-5 - Information Technology - Coding of Audio-Visual Objects - Part 5: Reference Software. ISO, 1999

1. ISO/IEC. JTC1/SC29/WG11, IS14496-2 - Information Technology - Coding of Audio-Visual A IEEE Trans. Commun., 33 (1985) 697-707.

A. Katsaggelos, L.P. Kondi, F.W. Meier, J. Ostermann, and G.M. Schuster, MPEG-4 and rate-distortionbased shape-coding techniques, Proc. IEEE, 86 (1998) 1126-1154.

25. A. Kaup, Object-based texture coding of moving video in MPEG-4, IEEE Trans. Circuits Syst. Video

Technol., 9 (1999) 5-15.
26. J.I. Kim, A.C. Bovik, and B.L. Evans, Generalized predictive binary shape coding using polygon approximation, Signal Process.: Image Commun., 15 (2000) 643-663.

8. M. Kunt, A. Ikonomopoulos, and Kocher, Second-generation image-coding techniques, Proc. IEEE,

9. J.-W. Lee, A. Vetro, Y. Wang, and Y.-S. Ho, Bit allocation for MPEG-4 video coding with spatio-
temporal trade-offs, IEEE Trans. Circuits Syst Video Technol., 13 (2003) 488-502. H.G. Musmann, M. IEE Trans. Circuits Syst. Video Technol, 13 (2003) 488-502.

Mages, Signal Proc Hotter, and J. Ostermann, Object-oriented analysis-synthesis coding of moving A.N. Netravali and B.G. Hage Commun., I (1989) 117-138.

New York, 1988.

32. D.L. Neuhoff and $K G$. Cas $A$ a ate and disortion analysis of chin codes for line dengs, IEEE Trans. Inf. Theory, 31 (1985) 53-67.

33. P. Nunes, F. Mar Fes, Fereira, and A Gasull, A contour-based approach to binary shape coding using multiple grid chain code, Signal Process.: Image Commun., 15 (2000) 585-600.

34. K.J. O'Connell, Object-adaptive vertex-based shape coding method, IEEE Trans. Circuits SY.st 7 (1997) $251-255$.

35. J. Ostermann, Efficient encoding of binary shapes using MPEG-4, Proceedings of IEEE International Conference on Image Processing, ICIP' 98 , Chicago, USA, October 1998

36. P. van Otterloo, A Contour-Oriented Approach for Shape Analysis, Prentice-Hall, Hertfordshire, UK, 1991.

37. T. Ozcelik and A.K. Katsaggelos, Very low bit rate video coding based on statistical spatiotemporal prediction of motion, segmentation and intensity fields, in Video Data Compression for Multimedia Computing, H.H. Li, S. Sun, and H. Derin, Eds., Kluwer, Norwell, MA, 1997, pp.

38. R. Prasad, J.W. Vieveen, J.H. Bons, and J.C. Arnbak, Relative vector probabilities in differential chain coded linedrawings, Proceedings of IEEE Pacific Rim Conference on Communication, Computers and .

J.A. Saghri and H. Freeman, Analysis of the precision of generalized chain codes for the representation of planar curves, IEEE Trans. Pattern Anal. Machine Intell., 3 (1981) 533-539.

. P. Salama and C. Huang, Error concealment for shape coding, Proceedings of IEEE International Conference on Image Processing, vol. 2, pp. 701-704, Rochester, USA, Sept. 2002.

. P. Salembier, F. Marques, and A. Gasull, Coding of partition sequences, in Video Coding, L. Torres and M. Kunt, Eds. Kluwer Academic Publishers, Englewood Cliffs. pp. 125-170, 1996.
43. S. Shirani, B. Erol. and F. Kossentini. A concealment method for shape information in MPEG-4 coded video sequences, IEEE Trans. Multimedia, 2 (2000) 185-190.

44. J. Signes. Binary format for scenes (BIFS): combining MPEG-4 media to build rich multimediat services, Proceedings of the Visual Communications and Image Processing. San Jose. CA. Jan. 1999.

L.D. Soares and F. Pereira. Refreshment need metrics for improved shape and texture object-based resilient video coding, IEEE Trans. Image Process., 12 (2003) 328-340.

6. L.D. Soares and F. Pereira, Spatial shape error concealment for object-based image and video coding. IEEE Trans. Image Process., 13 (2004) 586-599.

7. L.D. Soares and F. Pereira, Adaptive shape and texture intra refreshment schemes for improved error

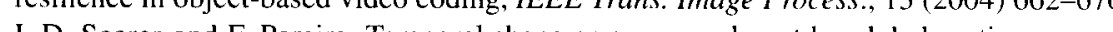

A Vetro T Haw K Sumi and $\mathrm{H}$ Sun Object-based coding for lon

video. Proceedings of IEEE International Conference on Multimedia and Expo, ICME'03, Beillance USA. July 2003.

. A. Vetro, H. Sun. and Y. Wang, MPEG-4 rate control for coding multiple video objects. IEEE Tran Circuits Syst. Video Technol., 9 (1999) 186-199.

1. A. Vetro, H. Sun, and Y. Wang, Object-based transcoding for adaptable video content delivery, IEEE

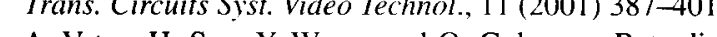

A. Vero, H. Sun, Y. Wang, and $O$. Gulyeruz, Rate-distortion modeling of binary shape using state

A. Vetro, $Y$ Wang, and

g, and H. Sun, Rate-distortion modeling for multiscale binary shape coding based on The

H. Wang, G.M. Schuster, A.K. Katsaggelos, and T.N. Pappas, An efficient rate-distortion optima 1181-1193. (1972) $269-281$. 